\title{
193 PERSONALITY HARDINESS, BURNOUT AND SPORT COMPETITION ANXIETY AMONG ATHLETICS AND WRESTLING COACHES
}

Harmanpreet Singh Bawa NIS, Patiala, Punjab, India

\subsection{6/bjsm.2010.078725.193}

Coaching is a challenging profession that requires the coaches to reconcile the often contrary goals of developing winning athletic programme and providing positive personal and academic experiences for their athletes. In at least all the games and sports, about $50 \%$ is the role of a coach to prepare a team/ athlete to give high performance in national and international competitions. The pressure to win, coming up to the expectation of the people and ever present interpersonal demands can lead to stress and render coaches susceptible to burnout. Persons with hard personality can easily and constructively cope with acute stress and burnout. Personality hardiness of an individual has relationships with his burnout and competitive trait anxiety. The coaches who are higher in personality hardiness are less susceptible to burnout. Personality hardiness is defined as multidimensional personality construct comprising three characteristics, control, commitment and challenge. Burnout refers to the loss of enthusiasm, excitement and essence of mission in one's work. It also causes feelings of helplessness, hopelessness, depression, meaninglessness, negative self concept and attitude towards work, life and other peoples. Burnout leads to emotional stress and physiological problems. Burnout is thought to be a consequence of prolonged exposure to stress and perception of an imbalance between situational demands and the resource available to meet these demands. Sport competition anxiety is another dimension related to sport performance hardness and burnout among athletes and coaches is quite a new topic in research on which researchers have started working in the last two decades. The investigator who has been national level cyclist, swimmer observed and felt lack of motivation, enthusiasm interest and dedication among coaches. The study was conducted with a purpose to determine the status of Indian athletics and wrestling coaches pertaining to their personality hardness, burnout and sport competition anxiety. The subjects of the present study were 30 athletes and 30 wrestlers. The short version of the hardness scale developed by Kobasa and Maddi (1982) was used to assess the hardiness of the subjects. The scale contains 36 items in all that assess the attitude of the subject on the three personality dimensions that is, commitment, control and challenge, Competitive trait anxiety was measured by administrating Sport Competition Anxiety Test by Rainer Marten (1986). Maslach burnout inventory (Maslach and Jackson 1986) was administered to assess the burnout status of the 
athletics and wrestling coaches. The inventory assesses the three aspects of burnout syndrome that is, emotional exhaustion, depersonalisation and lack of personal accomplishment. Coefficient of correlations were computed among various variables with regard to athletes and wrestlers, mean, SDs and ' $t$ ' values were computed in all variables between athletes and wrestlers. Significant correlation between emotional exhaustion and depersonalisation, between emotional exhaustion and competitive anxiety, between commitment and control, commitment and challenge, commitment and total scores of hardiness, control and total scores of hardiness, challenge and total scores of hardiness in case of athletic coaches and wrestling coaches were observed. Athletic coaches were found to be significantly younger, significantly greater in control disposition of personality hardiness and having significantly lesser amount of competition anxiety when compared with the wrestling coaches. 